\title{
Consideraciones geográficas en la interpretación de los resultados de revisiones sistemáticas y meta-análisis: fluidos en sepsis pediátrica
}

\section{Considering the geographical origin of studies when interpreting results from systematic reviews and meta-analyses: the case of fluid resuscitation in pediatric sepsis}

Franco Díaz $^{\mathrm{a}, \mathrm{b}, \mathrm{c}}$, Sebastián González-Dambrauskas ${ }^{\mathrm{a}, \mathrm{d}}$, Jan Hau Lee ${ }^{\mathrm{e}}$, Pablo Cruces $^{\mathrm{a}, \mathrm{f}}$

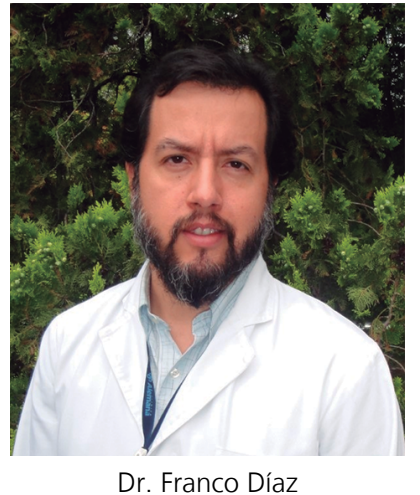

Dr. Franco Díaz

aRed Colaborativa Pediátrica de Latinoamérica (LARed Network)

bFacultad de Medicina Clínica Alemana Universidad del Desarrollo, Santiago, Chile

CUnidad de Paciente Crítico Pediátrico, Hospital Clínico La Florida Dra. Eloísa Díaz Insunza, Santiago, Chile

${ }^{d}$ Cuidados Intensivos Pediátricos Especializados, Casa de Galicia, Montevideo, Uruguay

'Children's Intensive Care Unit, KK Women's and Children's Hospital, Singapore

${ }^{\dagger}$ Centro de Investigación de Medicina Veterinaria, Facultad de Ciencias de la Vida, Universidad Andrés Bello, Santiago, Chile

El intercambio de conocimientos en todo el mundo se ha facilitado de una manera sin precedentes durante este siglo. La investigación científica y la literatura académica ha aumentado exponencialmente durante los últimos 20 años, rebosando la mayoría de los repositorios científicos y bases de datos. A modo de ejemplo, durante el 2017, aproximadamente 3.500 artículos fueron indexados por día en PubMed. Al restringir la búsqueda a publicaciones que tengan la palabra clave "pediatrics", aún arroja alrededor de 5.000 artículos por mes agregados en esa base de datos ${ }^{1}$.

Para lograr manejar esa abrumadora cantidad de información, las revisiones sistemáticas y los metaanálisis se han multiplicado y durante mucho tiempo han sido considerados como la información de mejor calidad en el enfoque de la medicina basada en la evidencia. Se sabe que las revisiones sistemáticas y los meta-análisis tienen deficiencias $\mathrm{y}$, a pesar de su abun- dancia, se estima que solo el 3\% son clínicamente relevantes ${ }^{2}$.

Muchos investigadores y grupos académicos han recurrido a nuevas metodologías, como los meta-análisis secuenciales de estudios prospectivos (trial sequential meta-analyses) y los meta-análisis acumulativos en red (cumulative network meta-analyses), para mejorar la calidad y la validez de las revisiones sistemáticas y los meta-análisis ${ }^{3}$. Se han encontrado sesgos sistemáticos en muchos aspectos, como la selección de bases de datos de búsqueda, conflictos de intereses con la industria y los autores, o la tendencia a incluir estudios si han sido publicados, que tuvieron un resultado estadísticamente positivo y con un gran efecto en el resultado clínico evaluado, entre otras ${ }^{2-4}$. Otro de estos sesgos podría ser el idioma. En algunas áreas de investigación se ha descrito que los estudios que no han sido escritos en inglés son más proclives a ser no considerados ${ }^{5}$.

Correspondencia:

Pablo Cruces

pcrucesr@gmail.com 
Recientemente, Alobaidi et al. publicaron una revisión sistemática y meta-análisis muy importante en JAMA pediatrics ${ }^{6}$. Realizaron una búsqueda y análisis exhaustivos de estudios sobre la sobrecarga de líquidos (FO) en niños críticamente enfermos. Encontraron 7.211 estudios potenciales, finalmente incluyeron 44 estudios (7.507 niños) en su análisis: 27 estudios de cohorte retrospectivos, 13 estudios de cohorte prospectivos, tres estudios de casos y controles y un ensayo clínico alesatorizado controlado. Concluyeron que FO se asoció con un mayor riesgo de mortalidad y otras morbilidades adquiridas durante la estadía en la Unidad de Cuidados Intensivos Pediátricos. Esta asociación se mostró utilizando varias definiciones de FO. Este artículo es muy relevante porque muestra a través de un enfoque sistemático y una bioestadística sólida, la asociación entre FO y morbimortalidad, un problema que afecta a muchos niños críticamente enfermos en todo el mundo ${ }^{7}$.

Revisamos los estudios incluidos en este meta-análisis y los marcamos en un mapa según la ubicación primaria dónde se desarrolló la investigación, utilizando el programa Tableau Desktop ${ }^{\circledR} 10.4$ (Tableau Soft- ware, Seattle, Washington, EE. UU.). Como se muestra en la Figura 1, la mayoría de los estudios provienen de países desarrollados [América del Norte y Europa (34, $77,3 \%)]$, y menos de una cuarta parte de los estudios $(10,22,7 \%)$ fueron de países de ingresos bajos y medios (LMIC).

Se puede argumentar que la investigación en LMIC es muy escasa, casi ausente, y la calidad de los estudios es baja. Reconocemos que existen muchas limitaciones para la investigación en regiones no desarrolladas ${ }^{8}$. Pero en estas mismas limitaciones radican muchos de los problemas responsables de la alta tasa de mortalidad infantil en estas áreas geográficas. Hay que considerar también que la realización de investigaciones y ensayos clínicos tiene una correlación moderada con la carga de enfermedad, especialmente en regiones con mayor pobreza'.

Debido a un sin número de razones, estudios con similares preguntas de investigación pueden tener resultados distintos al ser ejecutados en diferentes ubicaciones geográficas. Dos ensayos aleatorios sobre la reanimación con fluidos en shock séptico pediátrico, los estudios FEAST y FISH, son buenos ejemplos. En

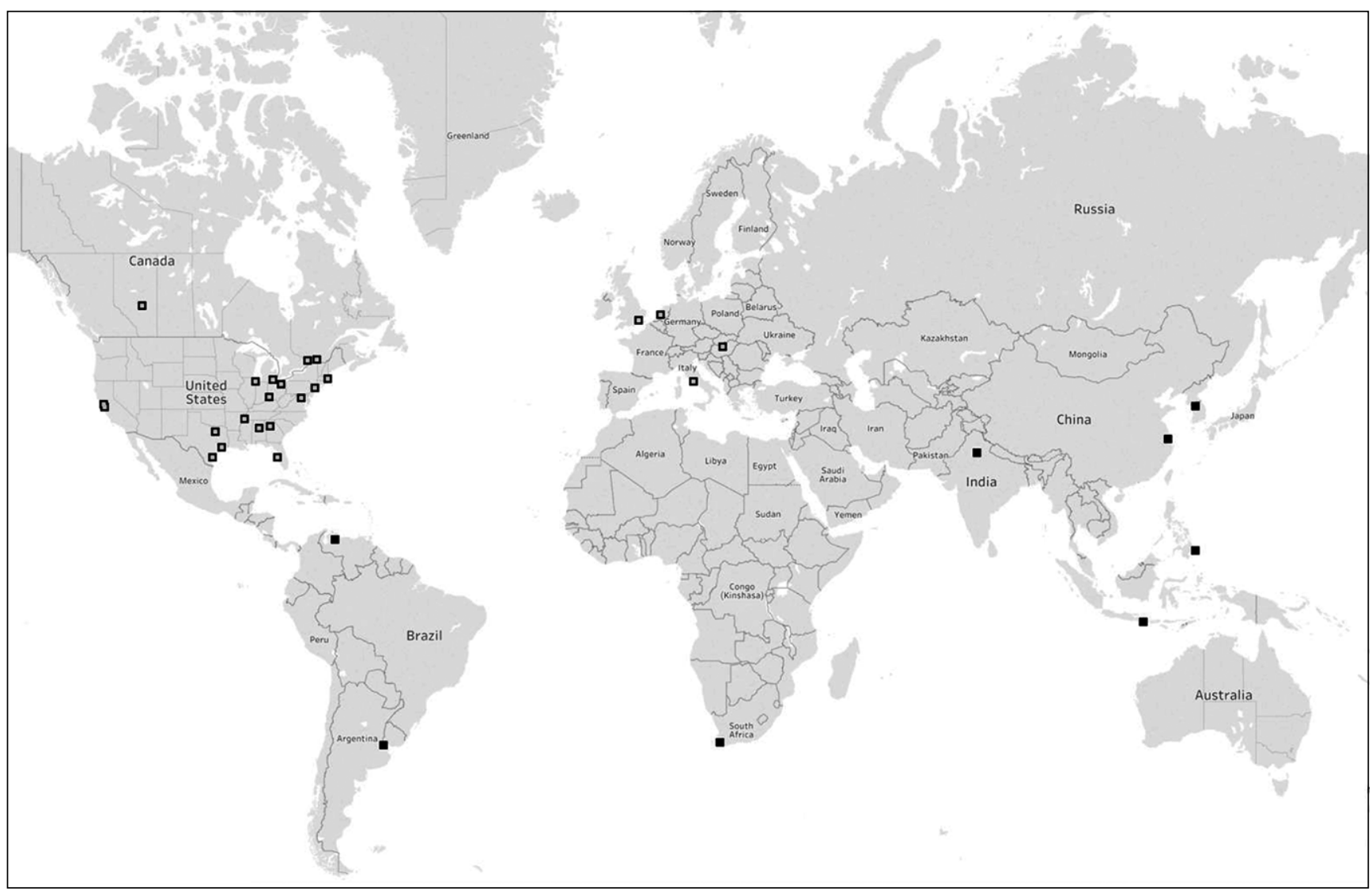

Figura 1. Mapa que muestra la ubicación de cada estudio incluido en la revisión sistemática y meta-análisis de Alobaidi R. et al ${ }^{6}$. Cada cuadrado representa la ciudad en que cada estudio fue realizado en forma primaria. Los cuadrados negros representan países de ingresos medio y bajo, mientras que los países desarrollados están representados por cuadrados grises. 
el ensayo FEAST, Maitland et al. mostró que los niños africanos con shock aleatorizados a reanimación rápida de fluidos (bolos de 20 a $40 \mathrm{ml} / \mathrm{kg}$ ) tenían un riesgo absoluto mayor de muerte de $3,3 \%$ a las 48 horas en comparación con los controles sin bolos ${ }^{10}$. Por otro lado, en el ensayo FISH, Inwald DP et al. no encontraron diferencias en los resultados de los niños con shock séptico en el Reino Unido aleatorizados a la estrategia restrictiva de bolos de fluido $(10 \mathrm{ml} / \mathrm{kg})$ en comparación con el tratamiento estándar $(20 \mathrm{ml} / \mathrm{kg})^{11}$.

El artículo de Alobaidi et al. aborda una pregunta relevante, porque los fluidos y la sepsis han sido un tema importante de debate en cuidados críticos en los últimos 10 años. Los LMIC estuvieron subrepresentados en esta revisión sistemática ${ }^{6}$. Desafortunadamente, esto es muy frecuente en la mayoría de los consensos, guías clínicas e incluso artículos que tratan sobre la salud global y la agenda de $\mathrm{LMIC}^{8,12,13}$.

Como profesionales dentro de las regiones LMIC, debemos preguntarnos cómo se pueden contextualizar los hallazgos y conclusiones de los meta-análisis y las revisiones sistemáticas a nuestra práctica diaria. Este tema es muy relevante, ya que las enfermedades infecciosas, especialmente en presencia de sepsis, son las principales causas de mortalidad infantil en el mun- do $^{14}$. Además de la etiología, hay otros factores que debemos considerar al comparar las regiones desarrolladas y LMIC. Esto incluye la oportunidad de acceso a la atención médica, la distancia de los servicios de salud, la duración de la enfermedad antes de buscar atención médica, así como los recursos humanos y tecnológicos disponibles en las unidades de cuidados intensivos pediátricos locales ${ }^{15}$.

Dadas estas disparidades en el mundo, debemos alentar a más discusiones que reconozcan las diferencias regionales en las recomendaciones, guías clínicas, definiciones de consenso y resultados de revisiones sistemáticas, como la que se discutió anteriormente, con el fin de mejorar los resultados clínicos de los niños críticamente enfermos. De hecho, es posible que un solo enfoque no se ajuste a todos en la prestación de cuidados críticos pediátricos en todo el mundo. Se necesitan más esfuerzos para hacer armonizar la carga de enfermedad con las poblaciones representativas y así acercar el punto de encuentro entre la evidencia y la práctica del mundo real.

\section{Conflicto de intereses}

Los autores declaran no tener conflicto de intereses.

\section{Referencias}

1. Alexandru Dan Corlan. Medline trend: automated yearly statistics of PubMed results for any query, 2004. Web resource at URL:http://dan.corlan.net/medlinetrend.html. Accessed: 2019-01-10.

2. Ioannidis JP. The Mass Production of Redundant, Misleading, and Conflicted Systematic Reviews and Meta-analyses. Milbank Q. 2016; 94(3):485-514.

3. Møller MH, Ioannidis JPA, Darmon M Are systematic reviews and meta-analyses still useful research? We are not sure. Intensive Care Med. 2018;44(4):518-20.

4. Chevret S, Ferguson ND, Bellomo R. Are systematic reviews and meta-analyses still useful research? No. Intensive Care Med. 2018;44(4):515-7.

5. Hartling L, Featherstone R, Nuspl M, Shave K, Dryden DM, Vandermeer B. Grey literature in systematic reviews: a cross-sectional study of the contribution of non-English reports, unpublished studies and dissertations to the results of meta-analyses in child-relevant reviews. BMC Med Res Methodol. 2017;17(1):64.

6. Alobaidi R, Morgan C, Basu RK, et al.
Association Between Fluid Balance and Outcomes in Critically Ill Children: A Systematic Review and Meta-analysis. JAMA Pediatr. 2018; 172(3):257-68.

7. Liu L, Oza S, Hogan D, et al. Global, regional, and national causes of under-5 mortality in 2000-15: an updated systematic analysis with implications for the Sustainable Development Goals. Lancet. 2016; 388(10063):3027-35.

8. von Saint André-von Arnim AO, Attebery J, Kortz TB, et al.; Pediatric Acute Lung Injury and Sepsis Investigators (PALISI) Network. Challenges and Priorities for Pediatric Critical Care ClinicianResearchers in Low- and Middle-Income Countries. Front Pediatr. 2017; 5:277.

9. Bourgeois FT, Olson KL, Ioannidis JP, Mandl KD. Association between pediatric clinical trials and global burden of disease. Pediatrics. 2014; 133(1):78-87.

10. Maitland K, Kiguli S, Opoka RO, et al. Mortality after fluid bolus in African children with severe infection. $\mathrm{N}$ Engl J Med. 2011; 364: 2483-2495.

11. Inwald DP, Canter R, Woolfall K, et al. PERUKI (Paediatric Emergency Research in the UK and Ireland) and PICS SG
(Paediatric Intensive Care Society Study Group). Restricted fluid bolus volume in early septic shock: results of the Fluids in Shock pilot trial. Arch Dis Child. 2018: Aug 7 [Epub ahead of print].

12. Slusher TM, Kiragu AW, Day LT, et al. Pediatric Critical Care in ResourceLimited Settings-Overview and Lessons Learned. Front Pediatr. 2018;6:49.

13. Pediatric Acute Lung Injury Consensus Conference Group. Pediatric acute respiratory distress syndrome: consensus recommendations from the Pediatric Acute Lung Injury Consensus Conference. Pediatr Crit Care Med. 2015;16(5):428-39.

14. Dugani S, Kissoon N. Global advocacy needed for sepsis in children. J Infect. 2017; Suppl 1:S61-5.

15. Diaz F, Carvajal C, GonzálezDambrauskas S, Serra A, MonteverdeFernández, N, Nuñez MJ, Cruces P. Organizational Characteristics and Resources In Latin-American Pediatric Intensive Care Units. Preliminary Report of REAL-CIP (Realidad En América Latina de Cuidados Intensivos Pediátricos) Study. Pediatr Crit Care Med. 19(6S):19. 